\title{
UNIFORM PRICE AUCTION OF DIVISIBLE GOODS BASED ON MULTIPLE ROUNDS LINEAR BIDDING AND ITS EQUILIBRIUM ANALYSIS
}

\author{
Congjun $\mathrm{RAO}^{\mathrm{a}, \mathrm{b}, \mathrm{c}}$, Yong $\mathrm{ZHAO}^{\mathrm{b}}$, Yang $\mathrm{CHEN}^{\mathrm{b}}$ \\ ${ }^{a}$ College of Mathematics and Computer Science, Huanggang Normal University, \\ 438000 Huanggang, P. R. China \\ ${ }^{\mathrm{b}}$ Institute of Systems Engineering, Huazhong University of Science and Technology, \\ 430074 Wuhan, P. R. China \\ 'School of Management, Huazhong University of Science and Technology, \\ 430074 Wuhan, P. R. China
}

Received 15 April 2012; accepted 06 August 2012

\begin{abstract}
In this paper, the auction problem of a kind of continuous homogeneous divisible goods is studied and a uniform price auction mechanism is presented based on three conditions, i.e. the auctioneer's supply is variable, every bidder submits multiple rounds continuous linear bidding, and every bidder's valuation to per unit of the goods is independent private information. Concretely, two key problems, i.e. the bidders' asymptotic strategic behaviours and forming process and composition of equilibrium points are explored. The conclusion is drawn that different bidders' bidding order and different starting points of initial bidding would not cause different local equilibrium points, and if the equilibrium points exist, then the equilibrium point is unique.
\end{abstract}

Keywords: divisible goods, uniform price auction, linear bidding, equilibrium points.

JEL Classification: D44, C02, C68, D61, D82.

\section{Introduction}

With the development of auction market of the emission rights, treasury and spectrum, multi-object auctions become one of the most active research areas in auction theory in recent years. Multi-object auctions can be classified as auctions of indivisible goods and auctions of divisible goods. In an auction of indivisible goods, the goods are indivisible, which means each good is an independent unit (e.g. auctions of mineral rights on federal land, offshore

Corresponding author Congjun Rao

E-mail: raocjun79@163.com 
drilling rights, procurement contracts, fish, flowers, wine, etc.). However, the goods in auctions of divisible goods are homogeneous and divisible, which means one unit good can be divided into many smaller units. For example, the auctions of emission rights, stocks, treasury bills, and spectrum are all the auctions of divisible goods (Rao, Zhao 2010). Nowadays, the divisible goods auctions have attracted increasing attention (Milgrom 2006; Rao, Peng 2009; Burinskiene, Rudzkis 2010; Kapliński, Tamošaitiene 2010). The divisible goods auction includes two different types, i.e. uniform price auction and discriminatory price auction.

In the last twenty years, considerable attention has been given to the use of auction by divisible goods with uniform price. Wilson (1979) was the first to consider uniform price auctions of divisible goods. He showed there is an equilibrium in which the asset is sold for half of its value in several settings. Klemperer (1989) generalized this result by showing that any low price can be supported in equilibrium. Based on these results, Back and Zender (1993) demonstrated how such a uniform-price auction can yield sensible results, consider the strategic difference between unit-demand and divisible goods auctions, and compare uniform-price and discriminatory auctions. This research has motivated further theoretical analysis of the divisible goods auction.

Since then, some theoretical literatures are emerged on how to reduce or eliminate the underpricing equilibrium in the Wilson/Back and Zender models. For example, Lengwiler (1999), Back and Zender (2001), McAdams (2002, 2007), LiCalzi and Pavan (2005), Rao and Zhao (2011a, b), Rao et al. (2010, 2012) showed that flexible supply can reduce underpricing. Moreover, Wang and Zender (2002) derived an equilibrium bidding strategy for a divisible goods auction involving asymmetrically informed risk-neutral and risk-averse bidders when there is random non-competitive demand. Later, Kremer and Nyborg (2004a, b) used a model of fixed supply divisible-good auctions, to study the effect of different rationing rules on the set of equilibrium prices. Chakraborty and Engelbrecht-Wiggan (2005) considered a uniform- price auction in which each of $n$ symmetric bidders has privately known, decreasing marginal values from an arbitrary $\mathrm{M}$-dimensional distribution and provided a quantile-type description of the asymptotic price that appropriately generalizes the characterization of the unit-demand asymptotic price. Mezzetti et al. (2008) studied sequential and single-round uniform-price auctions with affiliated values, and derive symmetric equilibrium for the auction in which $k_{1}$ objects are sold in the first round and $k_{2}$ in the second round, with and without revelation of the first-round winning bids. They demonstrated that auctioning objects in sequence generates a low balling effect that reduces the first-round price. Total revenue is greater in a single-round, uniform auction for $k=k_{1}+k_{2}$ objects than in a sequential uniform auction with no bid announcement.

Recently, scholars focused on the study on comparison between the uniform-price auction and the discriminatory auction. Bourjade (2009) modified the uniform-price auction rules in allowing the seller to ration bidders and proved that this rules provide a strategic foundation for underpricing when the seller has an interest in ownership dispersion and then many of the so-called "collusive-seeming" equilibria disappear. Brenner et al. (2009) investigated the revealed preferences of the issuers by surveying the sovereign issuers that conduct auctions and found that the majority of the issuers/countries in our sample use a discriminatory auction mechanism for issuing government debt, and they also concluded that market-oriented eco- 
nomies and those that practice common law tend to use a uniform method while economies who are less market oriented and practice civil law tend to use discriminatory price auctions. Holmberg (2009) characterized the Nash equilibrium in a pay-as-bid, divisible-good, procurement auction, and compared the offer curves and mark-ups of the derived equilibrium to the results for the SFE of a uniform-price auction. Genc (2009) compared the results for discriminatory auctions to results for uniform-price auctions when suppliers have capacity constraints. Damianov and Becker (2010), Damianov et al. (2010) examined an auction in which the seller determines the supply after observing the bids. They compare the uniform price and the discriminatory auction in a setting of supply uncertainty. Feng and Chatterjee (2010) studied a uniform-price auction, in which bidders demand no more than one item, and have independent, private values, and they showed that even when all buyers are present at the beginning of the auction, and when both the seller and the buyers are impatient, a sequential sale can still benefit the seller as it stimulates competition among forward-looking bidders. Jain and Walrand (2010) proposed a mechanism for auctioning bundles of multiple divisible goods in a network where buyers want the same amount of bandwidth on each link in their route. They showed that the proposed mechanism is a weak Nash implementation and there is Nash equilibrium of this auction game which yields the efficient allocation with strong budget balance. Rubchinsky (2010) considered the fair division problem for two participants in the presence of both divisible and indivisible items and designed a computationally efficient algorithm for finding all of them. Holmberg and Newbery (2010) presented an intuitive account of current understanding and showed how welfare losses depend on the number of firms in the market and their asymmetry. Sioshansi and Nicholson (2011) compared two types of uniform-price auction formats commonly used in wholesale electricity markets - centrally committed and self-committed markets. They derived Nash equilibria for both market designs in a symmetric duopoly setting, and also derived simple conditions under which the two market designs will be expected cost-equivalent. Groves (2011) studied a charity auction with multiple goods and showed that the highest-losing bid uniform price auction revenue dominates its lowest-winning bid counterpart.

These are all the important research results in uniform price auctions theory in the past few years. However, most of these results are obtained based on some simple and special conditions, such as unitary demand for every bidder's valuation follows uniform distribution, the bid price of bidders are discrete, the bidders are symmetrical and so on. When these conditions are changed, the equilibrium in uniform price auction is generally not unique. If the equilibrium is not unique, then we call this state as equilibrium excursion. Equilibrium excursion will certainly affect the auction proceeds, and is bad for estimating the allocative efficiency. Thus, mechanism design should not ignore to consider the change rules of equilibrium. Up to now, there is no literature to study the problem of equilibrium excursion, and to deal with how to induce the auction to a desired equilibrium state when there are multiple equilibriums in auction.

With the improvement of auctioneer's strategies as its main line, the elimination of low price equilibrium as its core, the induction of the ideal equilibrium as its keynote, this paper presents a uniform price auction mechanism with variable supply based on multiple rounds linear bidding. Different from the existing uniform price auction mechanisms, in 
our mechanism, all bidders are asymmetric and submit multiple rounds continuous linear bidding, the auctioneer's supply is variable, and every bidder's valuation to per unit of the goods is independent private information. Specially, we explore the bidders' asymptotic strategic behaviours and discuss the formation process of the desired equilibrium in theory, and then answer the question that whether the equilibrium excursion exists in our uniform price auction mechanism.

The rest of this paper is organized as follows. Section 1 gives some assumptions and definitions, and presents a uniform price auction mechanism with variable supply based on multiple rounds linear bidding. Section 2 analyzes the optimal bidding strategy of bidders. Section 3 discusses the forming process and composition of equilibrium points. Section 4 gives an auction example to show the forming process of equilibrium point. The final section concludes this paper.

\section{The auction mechanism}

First of all, we give some important assumptions and definitions. It is supposed that there is a risk neutral auctioneer who wants to auction $Q_{0}$ units of a continuous homogeneous divisible good. He faces $n(n>1)$ potential bidders, numbered $1,2, \cdots, n$. The set of bidders is denoted as $N=\{1,2, \cdots, n\}$. All bidders are risk neutral, and all want to maximize their expected profits. The per unit value of the goods to bidder $i$ is $v_{i}$ and is private information.

Moreover, we suppose that bidders are asymmetric, and each bidder submits a non-decreasing, piece-wise continuously differentiable demand function $q_{i}(p), i=1,2, \cdots, n$. Here, we set $q_{i}(p)$ is a continuous linear function $q_{i}(p)=a_{i}-b_{i} p$, which means his total demand at each price $p$, where $0 \leq q_{i}(p) \leq Q_{0}, a_{i} \geq 0, b_{i}>0$. The actual demand function of bidder $i$ is denoted as $q_{i}^{0}(p)=a_{i}^{0}-b_{i}^{0} p$ and is independent private information, where $a_{i}^{0} \geq 0, b_{i}^{0}>0$. Generally, the goal of each rational bidder is utility maximization, and the declared demand function $q_{i}(p)$ is less than or equal to the actual demand function $q_{i}^{0}(p)$.

Suppose that the auctioneer allocates goods under a uniform price. Concretely, the auctioneer strategically allocates $Q_{0}$ units of goods to $n$ bidders under a uniform price (market clearing price) $p^{*}$ after having observed the declared information of demand function $q_{i}(p), i=1,2, \cdots, n$. The allocated quantity for bidder $i$ is denoted as $q_{i}$, where $q_{i}=q_{i}\left(p^{*}\right)$. The maximum demand quantity of bidder $i$ is denoted as $G_{i}$. In auction, for the bidder $i$, he must pay $p^{*} q_{i}$ to get the quantity $q_{i}$, where satisfies $q_{i} \leq G_{i}$.

To improve the auctioneer's decision-making environment and increase the effectiveness of auction, we modify the fixed supply $\sum_{i=1}^{n} q_{i}=Q_{0}$ in the traditional auction models (Wilson 1979; Klemperer 1989; Back, Zender 1993) as the variable supply $\sum_{i=1}^{n} q_{i}=Q \leq Q_{0}$, which means the total supply $Q$ to the bidders before the auction is uncertain, where $Q \in\left[0, Q_{0}\right]$ is a value determined by the auctioneer after having observed the declared information of demand function $q_{i}(p)$. This information is common knowledge. 
The goal of the auctioneer is to maximize his income $p^{*} Q=\sum_{i=1}^{n} p^{*} q_{i}$ by choosing optimal supply $Q \leq Q_{0}$ and an optimal uniform price $p^{*}>0$. The auction model can be expressed by the following optimization problem $M_{1}$ :

$$
\begin{aligned}
& \operatorname{Max} U=p^{*} Q=p^{*} \sum_{i=1}^{n} q_{i}, \\
& \text { s.t. } \\
& \begin{cases}\sum_{i=1}^{n} q_{i}=Q \\
q_{i}=q_{i}\left(p^{*}\right)=a_{i}-b_{i} p^{*}, & i=1,2, \cdots, n \\
0 \leq q_{i} \leq G_{i}, & i=1,2, \cdots, n . \\
Q \leq Q_{0} & \end{cases}
\end{aligned}
$$

The goal of the $i^{\text {th }}$ bidder is to maximize his income by declaring his smart bidding strategy $q_{i}(p)$, that is:

$$
\begin{array}{cl}
\underset{q_{i}(p)}{\operatorname{Max}} & u_{i}=\left(v_{i}-p\right) q_{i}(p), \\
\text { s.t. } & q_{i}(p) \leq G_{i} .
\end{array}
$$

$M_{1}$ describes an auction mechanism of divisible goods under a uniform price with variable supply. For $M_{1}$, Rao and Zhao $(2010,2012)$ have proved his properties, i.e.: 1) This auction mechanism is a feasible auction mechanism, that is, it satisfy the individual rationality condition and incentive compatibility condition; 2) In equilibrium, the auctioneer's optimal supply strategy is $Q^{*}=Q_{0}$.

In the above auction mechanism, every bidder is allowed to submit multiple rounds bidding. In each round bidding, the auctioneer can let bidders submit their biddings according to a specific order (such as registration order). When all bidders complete the first round bidding, the auctioneer publishes the allocation results $\left(p^{1}, q_{1}^{1}, q_{2}^{1}, \cdots, q_{n}^{1}\right)$ in time, where $p^{1}$ is the uniform price when the first round bidding is over, and $q_{1}^{1}, q_{2}^{1}, \cdots, q_{n}^{1}$ denotes the allocated quantity for $n$ bidders. Based on these results, the bidders submit their second round biddings according to the same specific order. In the same way, the bidding process is carried on.

Suppose all bidders are completely rational, that is, from the second round bidding, each bidder chooses his optimal bidding strategy in this new round according to the allocation results of previous round biddings. His objective is to maximize his utility of current round bidding.

Specially, in order to reduce the arbitrary of bidding and improve the auction efficiency, all bidders' new biddings are not allowed to modify downward, i.e. if bidder $j$ submits his bidding $q_{j}^{(t)}(p)=a_{j}^{(t)}-b_{j}^{(t)} p$ in the $t^{\text {th }}$ round ( $t$ is a positive integer), and his new bidding in $(t+1)^{\text {th }}$ round is $q_{j}^{(t+1)}(p)=a_{j}^{(t+1)}-b_{j}^{(t+1)} p$, then the conditions $a_{j}^{(t+1)} \geq a_{j}^{(t)}, b_{j}^{(t+1)} \leq b_{j}^{(t)}$ must be satisfied. This bidding rule is called "Not allowed to modify downward rule". 
For the above auction mechanism, when the total supply $Q_{0}$ is full allocated to the bidders, i.e. $Q^{*}=Q_{0}$, and all bidders' incomes are no longer increased (all bidders are no longer to submit new biddings), the whole bidding process is over, and the auction reaches equilibrium state. Specially, the corresponding point $\left(p^{*}, q_{1}^{*}, q_{2}^{*}, \cdots, q_{n}^{*}\right)$ composed of equilibrium price $p^{*}$ and the allocated quantity $q_{i}^{*}(i=1,2, \cdots, n)$ is called equilibrium point.

In the following sections, we will explores the bidders' asymptotic strategic behaviours, and discuss the forming process of equilibrium points.

\section{Optimal bidding strategy analysis}

By the analysis of Section 1 , we know that the total supply $Q_{0}$ is full allocated to the bidders, i.e. $Q^{\star}=Q_{0}$ is a necessary condition to the equilibrium state of auction. Based on this necessary condition, we discuss the bidders' optimal bidding strategies and the forming process of equilibrium points.

Let the bidder $i$ 's bidding in the $t^{\text {th }}$ round be $q_{i}^{(t)}(p)=a_{i}^{(t)}-b_{i}^{(t)} p$, where $a_{i}^{(t)} \geq 0, b_{i}^{(t)}>0$, $i=1,2, \cdots, n$, and his new bidding in $(t+1)^{\text {th }}$ round be $q_{i}^{(t+1)}(p)=a_{i}^{(t+1)}-b_{i}^{(t+1)} p$, where $a_{i}^{(t+1)} \geq 0, b_{i}^{(t+1)}>0, i=1,2, \cdots, n$. In addition, all bidders' biddings must satisfy "Not allowed to modify downward rule", which means the conditions $a_{i}^{(t+1)} \geq a_{i}^{(t)}, b_{i}^{(t+1)} \leq b_{i}^{(t)}$ must be satisfied.

Proposition 1. Let bidder $i$ 's bidding in the $t^{\text {th }}$ round be $q_{i}^{(t)}(p)=a_{i}^{(t)}-b_{i}^{(t)} p$, where $a_{i}^{(t)} \geq 0, b_{i}^{(t)}>0, i=1,2, \cdots, n$, and $q_{i}^{(t)}(p) \leq G_{i}$, then we have:

(i) If the condition $\sum_{i=1}^{n} a_{i}^{(t)}<2 Q_{0}$ is satisfied, then the total supply $Q_{0}$ is not full allocated to the bidders, and the uniform price and the allocated quantity to bidder $i$ of current $t^{\text {th }}$ round bidding are $p^{(t)}=\frac{\sum_{i=1}^{n} a_{i}^{(t)}}{2 \sum_{i=1}^{n} b_{i}^{(t)}}$ and $q_{i}^{(t)}=a_{i}^{(t)}-\frac{b_{i}^{(t)} \sum_{i=1}^{n} a_{i}^{(t)}}{2 \sum_{i=1}^{n} b_{i}^{(t)}}, i=1,2, \cdots, n$, respectively;

(ii) If the condition $\sum_{i=1}^{n} a_{i}^{(t)}>2 Q_{0}$ is satisfied, and when there exists $k \in N$ such that $a_{k}^{(t)}-b_{k}^{(t)} \cdot \frac{\sum_{i=1}^{n} a_{i}^{(t)}-Q_{0}}{\sum_{i=1}^{n} b_{i}^{(t)}}>G_{k}$, then the total supply $Q_{0}$ is not full allocated to the bidders, and the uniform price of current $t^{\text {th }}$ round bidding is $p^{(t)}=\frac{a_{k}^{(t)}-G_{k}}{b_{k}^{(t)}}$, and the allocated quantity to bidder $k$ is $q_{k}^{(t)}=G_{k}$, and the allocated quantity to bidder $j$ is $q_{j}^{(t)}=a_{j}^{(t)}-b_{j}^{(t)} \cdot \frac{a_{k}^{(t)}-G_{k}}{b_{k}^{(t)}}$, $j=1,2, \cdots, n, \quad j \neq k$; 
(iii) If the condition $\sum_{i=1}^{n} a_{i}^{(t)}=2 Q_{0}$ is satisfied, or if the condition $\sum_{i=1}^{n} a_{i}^{(t)}>2 Q_{0}$ is satisfied, and for any $i \in N$ such that $a_{i}^{(t)}-b_{i}^{(t)} \cdot \frac{\sum_{i=1}^{n} a_{i}^{(t)}-Q_{0}}{\sum_{i=1}^{n} b_{i}^{(t)}} \leq G_{i}$, then the total supply $Q_{0}$ is full allocated to the bidders, and the uniform price and the allocated quantity to bidder $i$ of current $t^{\text {th }}$ round bidding are $p^{(t)}=\frac{\sum_{i=1}^{n} a_{i}^{(t)}-Q_{0}}{\sum_{i=1}^{n} b_{i}^{(t)}}$ and $q_{i}^{(t)}=a_{i}^{(t)}-\frac{b_{i}^{(t)}\left(\sum_{i=1}^{n} a_{i}^{(t)}-Q_{0}\right)}{\sum_{i=1}^{n} b_{i}^{(t)}}, i=1,2, \cdots, n$ respectively.

Proof. By model $M_{1}$, in the $t^{\text {th }}$ round bidding, when bidder $i$ submit the bidding $q_{i}^{(t)}(p)=a_{i}^{(t)}-b_{i}^{(t)} p, i=1,2, \cdots, n$, he will get the allocated quantity $q_{i}^{(t)}=a_{i}^{(t)}-b_{i}^{(t)} p^{(t)}$, $i=1,2, \cdots, n$, and $q_{i}^{(t)}(p) \leq G_{i}$. Substitute $q_{i}^{(t)}$ into the auctioneer's revenue function $U$, we can obtain the auctioneer's revenue in the $t^{\text {th }}$ round bidding as follows:

$$
U^{(t)}=p^{(t)} Q^{(t)}=p^{(t)}\left(\sum_{i=1}^{n} a_{i}^{(t)}-p^{(t)} \sum_{i=1}^{n} b_{i}^{(t)}\right)=p^{(t)} \sum_{i=1}^{n} a_{i}^{(t)}-\left(p^{(t)}\right)^{2} \sum_{i=1}^{n} b_{i}^{(t)} .
$$

In order to achieve maximum the auctioneer's revenue, the following condition must be satisfied:

$$
\frac{\partial U^{(t)}}{\partial p^{(t)}}=\frac{\partial}{\partial p^{(t)}}\left[p^{(t)} \sum_{i=1}^{n} a_{i}^{(t)}-\left(p^{(t)}\right)^{2} \sum_{i=1}^{n} b_{i}^{(t)}\right]=0 .
$$

Namely,

$$
\sum_{i=1}^{n} a_{i}^{(t)}-2 p^{(t)} \sum_{i=1}^{n} b_{i}^{(t)}=0 .
$$

Thus we get the uniform price of current $t^{\text {th }}$ round bidding is:

$$
p^{(t)}=\frac{\sum_{i=1}^{n} a_{i}^{(t)}}{2 \sum_{i=1}^{n} b_{i}^{(t)}}
$$

Substitute it into $q_{i}^{(t)}=a_{i}^{(t)}-b_{i}^{(t)} p^{(t)}$, then the allocated quantity to bidder $i$ is:

$$
q_{i}^{(t)}=a_{i}^{(t)}-\frac{b_{i}^{(t)} \sum_{i=1}^{n} a_{i}^{(t)}}{2 \sum_{i=1}^{n} b_{i}^{(t)}}, i=1,2, \cdots, n
$$

Therefore, by (2) and (3), the total allocated quantity of the $t^{\text {th }}$ round bidding to all bidders is:

$$
Q^{(t)}=\sum_{i=1}^{n} q_{i}^{(t)}=\sum_{i=1}^{n}\left(a_{i}^{(t)}-\frac{b_{i}^{(t)} \sum_{i=1}^{n} a_{i}^{(t)}}{2 \sum_{i=1}^{n} b_{i}^{(t)}}\right)=\sum_{i=1}^{n} a_{i}^{(t)}-\frac{\sum_{i=1}^{n} a_{i}^{(t)}}{2 \sum_{i=1}^{n} b_{i}^{(t)}} \cdot \sum_{i=1}^{n} b_{i}^{(t)}=\frac{\sum_{i=1}^{n} a_{i}^{(t)}}{2} .
$$


Based on this result, the problem can be divided into four special cases as follows.

1) When $\sum_{i=1}^{n} a_{i}^{(t)}<2 Q_{0}$, by (4), we have $Q^{(t)}=\frac{\sum_{i=1}^{n} a_{i}^{(t)}}{2}<Q_{0}$, which means the total supply $Q_{0}$ is not full allocated to the bidders, and the uniform price and the allocated quantity to bidder $i$ of current $t^{\text {th }}$ round bidding are the results expressed by (2) and (3);

2) When $\sum_{i=1}^{n} a_{i}^{(t)}=2 Q_{0}$, by (4), we have $Q^{(t)}=\frac{\sum_{i=1}^{n} a_{i}^{(t)}}{2}=Q_{0}$, which means the total supply $Q_{0}$ is full allocated to the bidders. Thus, $\sum_{i=1}^{n} a_{i}^{(t)}=2 Q_{0}$ is the critical point for whether the good is full allocated to the bidders or not. When the goods is full allocated, we have:

$$
\sum_{i=1}^{n} q_{i}^{(t)}=\sum_{i=1}^{n} a_{i}^{(t)}-p^{(t)} \sum_{i=1}^{n} b_{i}^{(t)}=Q_{0}
$$

$\sum_{i=1}^{n} a_{i}^{(t)}-Q_{0}$

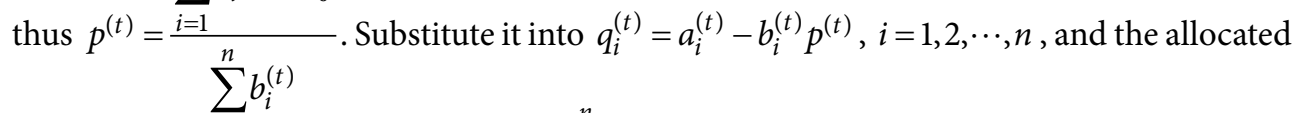

$$
\sum_{i=1}^{n} b_{i}^{(t)} b_{i}^{(t)}\left(\sum_{i=1}^{n} a_{i}^{(t)}-Q_{0}\right)
$$

quantity to bidder $i$ is $q_{i}^{(t)}=a_{i}^{(t)}-\frac{\sum_{i=1}^{n} a_{i=1}^{n} b_{i}^{(t)}}{\sum^{(t)}} i=1,2, \cdots, n$;

$$
\sum_{i=1}^{n} b_{i}^{(t)}
$$

3) When $\sum_{i=1}^{n} a_{i}^{(t)}>2 Q_{0}$, if for any $k \in N$ such that $a_{k}^{(t)}-b_{k}^{(t)} \cdot \frac{\sum_{i=1}^{n} a_{i}^{(t)}-Q_{0}}{\sum_{i=1}^{n} b_{i}^{(t)}}>G_{k}$, then the allocated quantity to bidder $i$ is $q_{k}^{(t)}=a_{k}^{(t)}-b_{k}^{(t)} p^{(t)}=G_{k}$, thus the uniform price is $p^{(t)}=\frac{a_{k}^{(t)}-G_{k}}{b_{k}^{(t)}}$, and the allocated quantity to bidder $j$ is $q_{j}^{(t)}=a_{j}^{(t)}-b_{j}^{(t)} \cdot \frac{a_{k}^{(t)}-G_{k}}{b_{k}^{(t)}}$, $j=1,2, \cdots, n$, and $j \neq k$.

$\sum^{n} a_{i}^{(t)}-Q_{0}$

By 2), when $p^{(t)}=\frac{\sum_{i=1}^{n}}{\sum_{i=1}^{n} b_{i}^{(t)}}$, the total allocated quantity of all bidders is

$$
\sum_{i=1}^{n} b_{i}^{(t)}
$$

$Q^{(t)}=\sum_{i=1}^{n}\left(a_{i}^{(t)}-b_{i}^{(t)} \cdot \frac{\sum_{i=1}^{n} a_{i}^{(t)}-Q_{0}}{\sum_{i=1}^{n} b_{i}^{(t)}}\right)=Q_{0}$. Thus, when $p^{(t)^{\prime}}=\frac{a_{k}^{(t)}-G_{k}}{b_{k}^{(t)}}>\frac{\sum_{i=1}^{n} a_{i}^{(t)}-Q_{0}}{\sum_{i=1}^{n} b_{i}^{(t)}}$, the total

allocated quantity of all bidders is $Q^{(t)^{\prime}}=\sum_{i=1}^{n}\left(a_{i}^{(t)}-b_{i}^{(t)} \cdot \frac{a_{k}^{(t)}-G_{k}}{b_{k}^{(t)}}\right)<Q_{0}$. 
4) When $\sum_{i=1}^{n} a_{i}^{(t)}>2 Q_{0}$, and for any $k \in N$ such that $a_{i}^{(t)}-b_{i}^{(t)} \cdot \frac{\sum_{i=1}^{n} a_{i}^{(t)}-Q_{0}}{\sum_{i=1}^{n} b_{i}^{(t)}} \leq G_{i}$, by (i), the uniform price must satisfy the condition $p^{(t)}=\leq \frac{\sum_{i=1}^{n} a_{i}(t)-Q_{0}}{\sum^{n}}$, so the total allocated quantity is:

$$
Q^{(t)}=\sum_{i=1}^{n}\left(a_{i}^{(t)}-b_{i}^{(t)} \cdot p^{(t)}\right) \geq \sum_{i=1}^{n}\left(a_{i}^{(t)}-b_{i}^{(t)} \cdot \frac{\sum_{i=1}^{n} a_{i}^{(t)}-Q_{0}}{\sum_{i=1}^{n} b_{i}^{(t)}}\right)=Q_{0} .
$$

Together with the maximal supply given by the auctioneer is $Q_{0}$, so we have $Q^{(t)}=Q_{0}$, which means $Q_{0}$ is full allocated to the bidders, and the uniform price and the allocated quantity to bidder $i$ of current $t^{\text {th }}$ round bidding are the same with case 2).

ByProposition 1, when $\sum_{i=1}^{n} a_{i}^{(t)} \geq 2 Q_{0}$, and for any $i \in N$ such that $a_{i}^{(t)}-b_{i}^{(t)} \cdot \frac{\sum_{i=1}^{n} a_{i}^{(t)}-Q_{0}}{\sum_{i=1}^{n} b_{i}^{(t)}} \leq G_{i}$, the total supply $Q_{0}$ is full allocated to the bidders. This result gives a sufficient condition for $Q_{0}$ is full allocated. This sufficient condition is denoted as C. Moreover, from above analysis, we know that $Q^{*}=Q_{0}$ is a necessary condition to the equilibrium state of auction. Thus, based on this necessary condition and condition $\mathrm{C}$, we discuss the bidders' optimal bidding strategies and the forming process of equilibrium points.

In addition, from Proposition 1, the following Corollary 1 can be deduced.

Corollary 1. For the above auction mechanism, if the bidder's bidding started from the $t^{\text {th }}$ round satisfies the condition $\mathrm{C}$, then the uniform price between two adjacent rounds satisfies $p^{(s+1)} \geq p^{(s)}$, and the auctioneer's is income between two adjacent rounds satisfies $U^{(s+1)} \geq U^{(s)}$, where $s \geq t$ ( $s$ and $t$ are all positive integers).

Proof. By Proposition 1, if the bidder's bidding started from the $t^{\text {th }}$ round satisfies the condition $\mathrm{C}$, then the total allocated quantity of the $s$ th round is $Q^{(s)}=Q_{0}$ for any $s \geq t$, and the corresponding uniform price is $p^{(s)}=\frac{\sum_{i=1}^{n} a_{i}^{(s)}-Q_{0}}{\sum_{i=1}^{n} b_{i}^{(s)}}$. Obviously, the value of $p^{(s)}$ increases with $a_{i}^{(s)}$ increasing, and $p^{(s)}$ increases with $b_{i}^{(s)}$ decreasing. Thus, when bidder $i(i \in N)$ submits his bidding of the $(t+1)^{\text {th }}$ round according to the rules $a_{i}^{(s+1)} \geq a_{i}^{(s)}, b_{i}^{(s+1)} \leq b_{i}^{(s)}$, the uniform price $p^{(s+1)}$ of the $(t+1)^{\text {th }}$ round must satisfy $p^{(s+1)} \geq p^{(s)}$. 
For the auctioneer, the incomes between two adjacent rounds are $U^{(s+1)}=p^{(s+1)} Q^{(s+1)}$ and $U^{(s)}=p^{(s)} Q^{(s)}$ respectively. Since $Q^{(s+1)}=Q^{(s)}=Q_{0}, p^{(s+1)} \geq p^{(s)}$, we have $U^{(s+1)} \geq U^{(s)}$.

From Proposition 1 and Corollary 1, we can conclude that in multiple rounds bidding process, all bidders' declared demand functions are gradually close to their actual demand functions, the uniform price is gradually increasing, the auctioneer's income is also gradually increasing. At the end, the $Q_{0}$ will be full allocated to the bidders. This results show that the auction mechanism given by this paper has good properties of information stimulant, and can effectively restrain tacit collusion and reduce many underpricing equilibrium. Moreover, in equilibrium, the auctioneer can realize his goal of utility maximization.

Based on Proposition 1 and Corollary 1, we discuss the bidders' optimal strategies.

Proposition 2. If the bidders' biddings after the $t^{\text {th }}$ round all satisfy the condition $\mathrm{C}$, then for the bidder $j(j=1,2, \cdots, n)$, we have:

$$
\left(a_{j}^{0}+\sum_{i=1}^{n} a_{i}^{(t)}\right)-Q_{0}
$$

1) If $a_{j}^{0}-b_{j}^{0} \cdot \frac{i \neq j}{b_{j}^{0}+\sum_{i=1}^{n} b_{i}^{(t)}}<G_{j}$, then bidder $j$ 's optimal strategy in the $(t+1)^{\text {th }}$ round $i \neq j$

bidding is to submit $q_{j}^{(t+1)}(p)=a_{j}^{0}-b_{j}^{0} p$, that is, he submits his actual demand function;

$$
\left(a_{j}^{0}+\sum_{i=1}^{n} a_{i}^{(t)}\right)-Q_{0}
$$

2) If $a_{j}^{0}-b_{j}^{0} \cdot \frac{i \neq j}{b_{j}^{0}+\sum_{\substack{i=1 \\ i \neq j}}^{n} b_{i}^{(t)}} \geq G_{j}$, then bidder $j$ 's optimal strategy in the $(t+1)^{\text {th }}$ round bidding is to submit $q_{j}^{(t+1)}(p)=a_{j}^{(t+1)}-b_{j}^{(t+1)} p$ according to the rule $a_{j}^{(t+1)} \geq a_{j}^{(t)}, b_{j}^{(t+1)} \leq b_{j}^{(t)}$, where $a_{j}^{(t+1)}, b_{j}^{(t+1)}$ satisfy the following condition:

$$
a_{j}^{(t+1)}-b_{j}^{(t+1)} \cdot \frac{\left(a_{j}^{(t+1)}+\sum_{\substack{i=1 \\ i \neq j}}^{n} a_{i}^{(t)}\right)-Q_{0}}{b_{j}^{(t+1)}+\sum_{\substack{i=1 \\ i \neq j}}^{n} b_{i}^{(t)}}=G_{j} .
$$

Proof. By Proposition 1, if the bidders' biddings after the $t^{\text {th }}$ round all satisfy the condition C, then the total allocated quantity of all bidders in the $t^{\text {th }}$ round bidding is $Q^{(t)}=Q_{0}$. After the bidder $j$ submit his new bidding $q_{j}^{(t+1)}(p)=a_{j}^{(t+1)}-b_{j}^{(t+1)} p$ in the $(t+1)^{\text {th }}$ round bidding according to the rule $a_{j}^{(t+1)} \geq a_{j}^{(t)}, b_{j}^{(t+1)} \leq b_{j}^{(t)}$, by Proposition 1 , the total allocated quantity in the $(t+1)^{\text {th }}$ round bidding is also $Q^{(t+1)}=Q_{0}$. Thus the uniform price of the $t^{\text {th }}$ round 


$$
\left(a_{j}^{(t+1)}+\sum_{i=1}^{n} a_{i}^{(t)}\right)-Q_{0}
$$

bidding is $p^{(t+1)}=\frac{i \neq j}{b_{j}^{(t+1)}+\sum_{i=1}^{n} b_{i}^{(t)}}$, and the bidder $j$ 's income can be expressed by:

$$
u_{j}^{(t+1)}=\left(v_{j}-p^{(t+1)}\right) q_{j}^{(t+1)} .
$$

Together with $q_{j}^{(t+1)}=a_{j}^{(t+1)}-b_{j}^{(t+1)} p^{(t+1)}$, so $u_{j}^{(t+1)}$ can be rewritten as:

$$
u_{j}^{(t+1)}=\left(v_{j}-\frac{a_{j}^{(t+1)}-q_{j}^{(t+1)}}{b_{j}^{(t+1)}}\right) q_{j}^{(t+1)}=v_{j} q_{j}^{(t+1)}-\frac{a_{j}^{(t+1)} q_{j}^{(t+1)}}{b_{j}^{(t+1)}}+\frac{\left(q_{j}^{(t+1)}\right)^{2}}{b_{j}^{(t+1)}} .
$$

So we have:

$$
\frac{\partial u_{j}^{(t+1)}}{\partial q_{j}^{(t+1)}}=v_{j}-\frac{a_{j}^{(t+1)}}{b_{j}^{(t+1)}}+\frac{2 q_{j}^{(t+1)}}{b_{j}^{(t+1)}} .
$$

Moreover, bidder $j$ 's actual demand function is $q_{j}^{0}(p)=a_{j}^{0}-b_{j}^{0} p$, so the possible maximal uniform price is $p_{\max }=\frac{a_{j}^{0}}{b_{j}^{0}}$. The goal of the $j^{\text {th }}$ bidder is to maximize his income by declaring his smart bidding strategy $q_{j}(p)$, that is, $\underset{q_{j}(p)}{\operatorname{Max}} u_{j}=\left(v_{j}-p\right) q_{j}(p),(1)$ is satisfied, so bidder $j$ 's $j(j \in N)$ valuation $v_{j}$ for per unit good satisfies $v_{j}-p_{\max }>0$, i.e. $v_{j}>\frac{a_{j}^{0}}{b_{j}^{0}}$. Moreover, by the "Not allowed to modify downward rule" $a_{i}^{(t+1)} \geq a_{i}^{(t)}, b_{i}^{(t+1)} \leq b_{i}^{(t)}$, and the assumption in Section 1 (the declared demand function $q_{i}(p)$ is less than or equal to the actual demand function $\left.q_{i}^{0}(p)\right)$, then we have $\frac{a_{j}^{0}}{b_{j}^{0}} \geq \frac{a_{j}^{(t+1)}}{b_{j}^{(t+1)}}$, so $v_{j}>\frac{a_{j}^{0}}{b_{j}^{0}} \geq \frac{a_{j}^{(t+1)}}{b_{j}^{(t+1)}}$. Together with (5), thus we have $\frac{\partial u_{j}^{(t+1)}}{\partial q_{j}^{(t+1)}}>0$, which means bidder $j^{\prime}$ 's income increases with $q_{j}^{(t+1)}$ increasing.

$$
\left(a_{j}^{(t+1)}+\sum_{i=1}^{n} a_{i}^{(t)}\right)-Q_{0}
$$

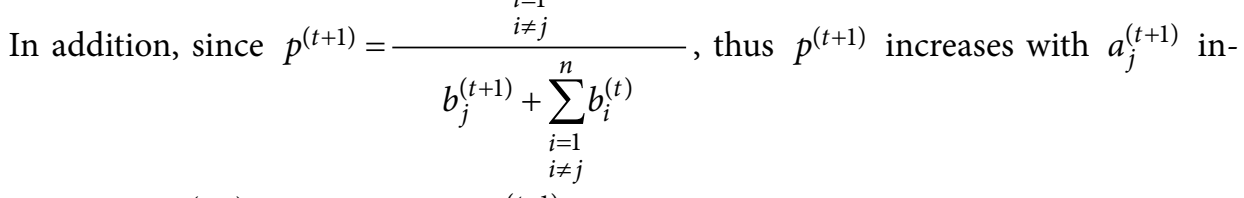
creasing, and $p^{(t+1)}$ increases with $b_{j}^{(t+1)}$ decreasing. The bidding strategies of the rest $n-1$ bidders are unchanged besides bidder $j$, so the rest $n-1$ bidders' allocated quantities decrease with $a_{j}^{(t+1)}$ increasing and decrease with $b_{j}^{(t+1)}$ decreasing. Therefore, bidder $j$ 's allocated quantity $q_{j}^{(t+1)}$ in the $(t+1)^{\text {th }}$ round bidding increases with $a_{j}^{(t+1)}$ increasing and increases with $b_{j}^{(t+1)}$ decreasing. 


$$
\text { Based on above analysis, when the condition } 0<a_{j}^{0}-b_{j}^{0} \cdot \frac{\left(a_{j}^{0}+\sum_{\substack{i=1 \\ i \neq j}}^{n} a_{i}^{(t)}\right)-Q_{0}}{b_{j}^{0}+\sum_{\substack{i=1 \\ i \neq j}}^{n} b_{i}^{(t)}}<G_{j} \text { is satisfied, }
$$

if and only if bidder $j(j \in N)$ submit the new bidding $q_{j}^{(t+1)}(p)=a_{j}^{(0)}-b_{j}^{(0)} p$ in the $(t+1)^{\text {th }}$

round bidding, bidder $j$ can get maximum allocated
and he can realize the goal of income maximization.
\[ a_{j}^{(0)}+\sum_{j=1}^{n} a_{i}^{(t)} \]

$$
\left(a_{j}^{0}+\sum_{i=1}^{n} a_{i}^{(t)}\right)-Q_{0}
$$

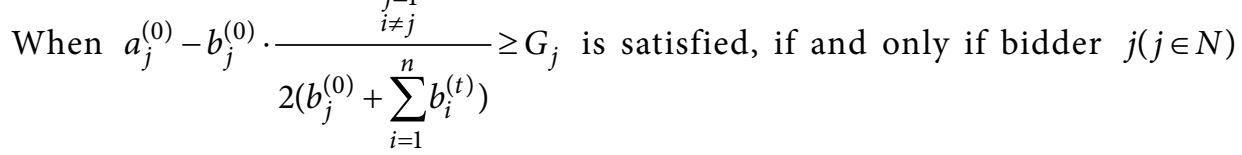
$i \neq j$

submit the new bidding $q_{j}^{(t+1)}(p)=a_{j}^{(t+1)}-b_{j}^{(t+1)} p$ which satisfies condition $a_{j}^{(t+1)}-$ $b_{j}^{(t+1)} \cdot \frac{\left(a_{j}^{(t+1)}+\sum_{\substack{i=1 \\ i \neq j}}^{n} a_{i}^{(t)}\right)-Q_{0}}{b_{j}^{(t+1)}+\sum_{i=1}^{n} b_{i}^{(t)}}=G_{j}$ in the $(t+1)^{\text {th }}$ round bidding, bidder $j$ can get maximum allocated quantity $G_{j}$, and his income reaches maximum value. Otherwise, if $a_{j}^{(t+1)}$ continues to increase, or $b_{j}^{(t+1)}$ continues to decrease, then the uniform price continues to increase, but his allocated quantity is still $q_{j}^{(t+1)}=G_{j}$, thus his income $u_{j}^{(t+1)}=\left(v_{j}-p^{(t+1)}\right) G_{j}$ will decrease.

\section{Forming process and composition analysis of equilibrium points}

In practical bidding process, when the first round bidding is over, the auctioneer can publish the allocation results in time by using model $M_{1}$. Start with the second round bidding, all bidders submit their biddings according to a specific order. Based on current allocation results, the bidders submit their next round biddings according to the optimal strategy given by Proposition 2 . When the total supply $Q_{0}$ is full allocated to the bidders, and all bidders' incomes are no longer increased, and the auction reaches equilibrium state, the whole bidding process is over.

Based on Proposition 1 and Proposition 2, next we discuss the forming process and composition of equilibrium point $\left(p^{*}, q_{1}^{*}, q_{2}^{*}, \cdots, q_{n}^{*}\right)$. 
Case 1: After the first round bidding, if all bidders' biddings reach the limit state, that is, they all declare their actual demand functions, $q_{j}^{(1)}(p)=a_{j}^{0}-b_{j}^{0} p, j=1,2, \cdots, n$, and the total allocated quantity is $Q^{*}=Q_{0}$, then the auction reaches equilibrium state. By Proposition 1 , the equilibrium point is as follows:

$$
\begin{gathered}
p^{*}=\frac{\sum_{i=1}^{n} a_{i}^{0}-Q_{0}}{\sum_{i=1}^{n} b_{i}^{0}} ; \\
q_{j}^{*}=a_{j}^{(0)}-\frac{b_{j}^{(0)}\left(\sum_{i=1}^{n} a_{i}^{0}-Q_{0}\right)}{\sum_{i=1}^{n} b_{i}^{0}}, j=1,2, \cdots, n .
\end{gathered}
$$

Case 2: After the first round bidding, if existing there several bidders' biddings (bidder $\left.k_{1}, k_{2}, \cdots, k_{h}, h \leq n\right)$ do not reach the limit state, this $h$ bidders will participate in the second or multiple rounds biddings.

Suppose that from the $t^{\text {th }}$ round bidding, all bidders' biddings of each round all satisfy the condition $C$. So in each round bidding from the $t^{\text {th }}$ round, the total allocated quantity $Q_{0}$ will be full allocated to the bidders, and each bidder will submit his bidding according to the optimal strategies given by Proposition 2 .

After $r(r \geq 2)$ rounds biddings, suppose that all bidders' biddings reach the limit state (they all declare their actual demand functions, i.e. $q_{j}^{(r)}(p)=a_{j}^{0}-b_{j}^{0} p$, or his bidding such that his allocated quantity reach maximal, that is, his declared bidding $q_{j}^{(r)}(p)=a_{j}^{(r)}-b_{j}^{(r)} p$ satisfies the condition $a_{j}^{(r)}-b_{j}^{(r)} \cdot \frac{\sum_{i=1}^{n} a_{i}^{(r)}-Q_{0}}{\sum_{i=1}^{n} b_{i}^{(r)}}=G_{j}$, then the auction reaches equilibrium state, and the equilibrium point is:

$$
p^{*}=\frac{\sum_{i=1}^{n} a_{i}^{(r)}-Q_{0}}{\sum_{i=1}^{n} b_{i}^{(r)}}
$$

$$
q_{j}^{*}=\left\{\begin{array}{cc}
G_{j}, \quad a_{j}^{(0)}-\frac{b_{j}^{(0)}\left(\sum_{i=1}^{n} a_{i}^{(r)}-Q_{0}\right)}{\sum_{i=1}^{n} b_{i}^{(r)}} \geq G_{j} \\
a_{j}^{(0)}-\frac{b_{j}^{(0)}\left(\sum_{i=1}^{n} a_{i}^{(r)}-Q_{0}\right)}{\sum_{i=1}^{n} b_{i}^{(r)}}, & a_{j}^{(0)}-\frac{b_{j}^{(0)}\left(\sum_{i=1}^{n} a_{i}^{(r)}-Q_{0}\right)}{\sum_{i=1}^{n} b_{i}^{(r)}}<G_{j}
\end{array}, j=1,2, \cdots, n .\right.
$$


Based on above conclusions, we get the following Proposition 3.

Proposition 3. For the above auction mechanism, the different bidders' bidding order and different starting points of initial bidding would not cause different local equilibrium points, and if the equilibrium points exist, then the equilibrium point is unique.

Proof: From above discussion, if the auction reaches equilibrium state after the first round bidding, then the equilibrium point is $p^{*}=\frac{\sum_{i=1}^{n} a_{i}^{(0)}-Q_{0}}{\sum_{i=1}^{n} b_{i}^{(0)}}$ and $q_{j}^{*}=a_{j}^{(0)}-\frac{b_{j}^{(0)}\left(\sum_{i=1}^{n} a_{i}^{(0)}-Q_{0}\right)}{\sum_{i=1}^{n} b_{i}^{(0)}}$. Obviously, from this results of $p^{*}$ and $q_{j}^{*}$, together with $a_{i}^{(0)}$ and $b_{i}^{(0)}, i=1,2, \cdots, n$ are all constants, we can see that the equilibrium point is unique, and the different bidders' bidding order and different starting points of initial bidding would not cause different local equilibrium points.

After $r(r \geq 2)$ rounds biddings, if all bidders will no longer submit new biddings, and $Q_{0}$ is $\sum_{i=1}^{n} a_{i}^{(r)}-Q_{0}$ full allocated to the bidders, then the equilibrium price is $p^{*}=\frac{\sum_{i=1}}{n}$, and the allocated quantity to bidder $j$ is $q_{j}^{*}=a_{j}^{(0)}-\frac{b_{j}^{(0)}\left(\sum_{i=1}^{n} a_{i}^{(r)}-Q_{0}\right)}{\sum_{i=1}^{n} b_{i}^{(r)}}$, or $q_{j}^{*}=G_{j}, j=1,2, \cdots, n$. Based on this

result, if we can prove that the uniform price $p^{*}$ in equilibrium is unique, then all bidders' allocated quantities $q_{1}^{*}, q_{2}^{*}, \cdots, q_{n}^{*}$ are all unique, thus the equilibrium point $\left(p^{*}, q_{1}^{*}, q_{2}^{*}, \cdots, q_{n}^{*}\right)$ is unique. Next we prove that the uniform price $p^{*}$ is unique.

In equilibrium, all bidders' bidding can be divided into two cases, i.e. they declare their actual demand function $q_{j}^{(r)}(p)=a_{j}^{0}-b_{j}^{0} p$, or submit the biddings such that their allocated quantities reach maximal, that is, the declared bidding $q_{j}^{(r)}(p)=a_{j}^{(r)}-b_{j}^{(r)} p$ satisfies the condition $a_{j}^{(r)}-b_{j}^{(r)} \cdot \frac{\sum_{i=1}^{n} a_{i}^{(r)}-Q_{0}}{\sum_{i=1}^{n} b_{i}^{(r)}}=G_{j}$. So in the equilibrium price $p^{*}=\frac{\sum_{i=1}^{n} a_{i}^{(r)}-Q_{0}}{\sum_{i=1}^{n} b_{i}^{(r)}}$, the values of part $a_{i}^{(r)}$ and $b_{i}^{(r)}$ (we set $\left.i=i_{1}, i_{2}, \cdots, i_{v}\right)$ are $a_{i}^{0}$ and $b_{i}^{0}$, thus $\sum_{i=i_{1}}^{i_{v}} a_{i}^{(r)}$ and $\sum_{i=i_{1}}^{i_{v}} b_{i}^{(r)}$ all are constant values. The values of other $a_{i}^{(r)}$ and $b_{i}^{(r)}$ (we set $i=i_{v+1}, i_{v+2}, \cdots, i_{n}$ ) must satisfy the condition $a_{i}^{(r)}-b_{i}^{(r)} \cdot \frac{\sum_{i=1}^{n} a_{i}^{(r)}-Q_{0}}{\sum^{n} b_{i}^{(r)}}=G_{i}$. Therefore, to prove the equilibrium price $\sum_{i=1}^{n} b_{i}^{(r)}$ 
110 C. Rao et al. Uniform price auction of divisible goods based on multiple rounds linear bidding ... $p^{*}=\frac{\sum_{i=1}^{n} a_{i}^{(r)}-Q_{0}}{\sum_{i=1}^{n} b_{i}^{(r)}}$ is a constant value, we only to prove that for any two bidding of bidder $i_{k}(k=v+1, v+2, \cdots, n), \quad q_{i_{k}}^{(r)}(p)=a_{i_{k}}^{(r)}-b_{i_{k}}^{(r)} p$ and $q_{i_{k}}^{(r)^{\prime}}(p)=a_{i_{k}}^{(r)^{\prime}}-b_{i_{k}}^{(r)^{\prime}} p$, where satisfy $a_{i_{k}}^{(r)}-b_{i_{k}}^{(r)} \cdot \frac{\sum_{i=1}^{n} a_{i}^{(r)}-Q_{0}}{\sum_{i=1}^{n} b_{i}^{(r)}}=G_{i_{k}}$ and $a_{i_{k}}^{(r)^{\prime}}-b_{i_{k}}^{(r)} \cdot \frac{\sum_{i=1}^{n} a_{i}^{(r)}-Q_{0}}{\sum_{i=1}^{n} b_{i}^{(r)}}=G_{i_{k}}$, the equilibrium price $a_{i_{k}}^{(r)}+\sum_{i=1}^{n} a_{i}^{(r)}-Q_{0} \quad a_{i_{k}}^{(r)^{\prime}}+\sum_{i=1}^{n} a_{i}^{(r)}-Q_{0}$ which we get are the same, i.e.

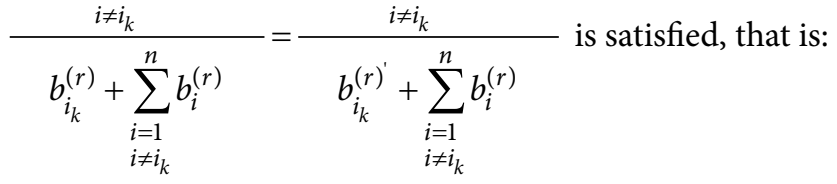

$$
a_{i_{k}}^{(r)^{\prime}} b_{i_{k}}^{(r)}+a_{i_{k}}^{(r)^{\prime}} \sum_{\substack{i=1 \\ i \neq i_{k}}}^{n} b_{i}^{(r)}+b_{i_{k}}^{(r)}\left(\sum_{\substack{i=1 \\ i \neq i_{k}}}^{n} a_{i}^{(r)}-Q_{0}\right)=a_{i_{k}}^{(r)} b_{i_{k}}^{(r)^{\prime}}+a_{i_{k}}^{(r)} \sum_{\substack{i=1 \\ i \neq i_{k}}}^{n} b_{i}^{(r)}+b_{i_{k}}^{(r)}\left(\sum_{\substack{i=1 \\ i \neq i_{k}}}^{n} a_{i}^{(r)}-Q_{0}\right) .
$$

In fact, since:

$$
a_{i_{k}}^{(r)}-b_{i_{k}}^{(r)} \cdot \frac{\sum_{i=1}^{n} a_{i}^{(r)}-Q_{0}}{\sum_{i=1}^{n} b_{i}^{(r)}}=a_{i_{k}}^{(r)}-b_{i_{k}}^{(r)^{\prime}} \cdot \frac{\sum_{i=1}^{n} a_{i}^{(r)}-Q_{0}}{\sum_{i=1}^{n} b_{i}^{(r)}}=G_{i_{k}},
$$

thus:

$$
a_{i_{k}}^{(r)^{\prime}} b_{i_{k}}^{(r)}+a_{i_{k}}^{(r)^{\prime}} \sum_{\substack{i=1 \\ i \neq i_{k}}}^{n} b_{i}^{(r)}-b_{i_{k}}^{(r)^{\prime}}\left(\sum_{\substack{i=1 \\ i \neq i_{k}}}^{n} a_{i}^{(r)}-Q_{0}\right)=a_{i_{k}}^{(r)} b_{i_{k}}^{(r)^{\prime}}+a_{i_{k}}^{(r)} \sum_{\substack{i=1 \\ i \neq i_{k}}}^{n} b_{i}^{(r)}-b_{i_{k}}^{(r)}\left(\sum_{\substack{i=1 \\ i \neq i_{k}}}^{n} a_{i}^{(r)}-Q_{0}\right) .
$$

So we have:

$$
a_{i_{k}}^{(r)} b_{i_{k}}^{(r)}+a_{i_{k}}^{(r)} \sum_{\substack{i=1 \\ i \neq i_{k}}}^{n} b_{i}^{(r)}+b_{i_{k}}^{(r)}\left(\sum_{\substack{i=1 \\ i \neq i_{k}}}^{n} a_{i}^{(r)}-Q_{0}\right)=a_{i_{k}}^{(r)} b_{i_{k}}^{(r)^{\prime}}+a_{i_{k}}^{(r)} \sum_{\substack{i=1 \\ i \neq i_{k}}}^{n} b_{i}^{(r)}+b_{i_{k}}^{(r)}\left(\sum_{\substack{i=1 \\ i \neq i_{k}}}^{n} a_{i}^{(r)}-Q_{0}\right) .
$$

For the bidder $j$, no matter which starting point of initial bidding he chooses, his declared bidding $q_{j}^{(r)}(p)=a_{j}^{(r)}-b_{j}^{(r)} p$ must satisfies the condition $a_{j}^{(r)}-b_{j}^{(r)} \cdot \frac{\sum_{i=1}^{n} a_{i}^{(r)}-Q_{0}}{\sum_{i=1}^{n} b_{i}^{(r)}}=G_{j}$ 
when the auction reaches equilibrium state, and the equilibrium point is a unique constant $p^{*}=\frac{\sum_{i=1}^{n} a_{i}^{(r)}-Q_{0}}{\sum_{i=1}^{n} b_{i}^{(r)}}$, which means the different starting points of initial bidding would not cause different local equilibrium points. In addition, for the different bidders' bidding order, the values of $\sum_{i=1}^{n} a_{i}^{(r)}$ and $\sum_{i=1}^{n} b_{i}^{(r)}$ will not change, so the value of $p^{*}=\frac{\sum_{i=1}^{n} a_{i}^{(r)}-Q_{0}}{\sum_{i=1}^{n} b_{i}^{(r)}}$ will not change, which means different bidders' bidding order would not cause different local equilibrium points.

From above proof, we can conclude that the different bidders' bidding order and different starting points of initial bidding would not cause different local equilibrium points, and if the equilibrium points exist, then the equilibrium point is unique.

In essence, in multiple rounds bidding, the bidders submit their biddings according to a specific order (they don't simultaneously submit their biddings), and all bidders know the former biddings before they submit the next round biddings, thus the auction process can be regarded as a complete information dynamic game. By the standard conclusion of the game theory: generally speaking, in the limited game with complete information, the equilibrium point is unique. This conclusion can be deduced by Backward Induction. From this, it can be seen that, the conclusion given by Proposition 3 is in accordance with the standard conclusion of the game theory. Thus, the conclusion given by Proposition 3 is reasonable.

\section{Description of auction process}

Based on the discussions and analysis in sections 1, 2 and 3, we give the following steps to show how to implement the uniform price auction of divisible goods based on multiple rounds linear bidding.

Step 1: The buyer publishes the basic requirement about the auction, and announces the bidding rules, for example, each bidder must submits a non-decreasing continuous linear function $q_{i}(p)=a_{i}-b_{i} p, i=1,2, \cdots, n$, every bidder is allowed to submit multiple rounds biddings, and the auctioneer can let bidders submit their biddings according to a specific order (such as registration order) in each round bidding, all bidders' new biddings must satisfy the "Not allowed to modify downward rule", and so on.

Step 2: The bidders submit their biddings to the auctioneer according to the basic requirement and bidding rules.

Step 3: Substituting the bidders' bidding data into model $M_{1}$. If all bidders' biddings reach the limit state after the first round bidding, i.e. they all declare their actual demand functions, $q_{j}^{(1)}(p)=a_{j}^{0}-b_{j}^{0} p, j=1,2, \cdots, n$, and the total allocated quantity is $Q^{*}=Q_{0}$, then the auction reaches equilibrium state, the auction is over, and the equilibrium price is $p^{*}$ which can be 
112 C. Rao et al. Uniform price auction of divisible goods based on multiple rounds linear bidding ...

computed by (6), the allocated quantity for bidder $j$ is $q_{j}^{*}$ which can be computed by (7). The bidder $j$ must pay $p^{*} q_{j}^{*}$ to get the quantity $q_{j}^{*}$.

Step 4: If existing there several bidders' biddings (bidder $k_{1}, k_{2}, \cdots, k_{h}, h \leq n$ ) do not reach the limit state after the first round bidding, this $h$ bidders will participate in the second or multiple rounds biddings. The detail bidding process is described as Case 2 in Section 4 . When all suppliers are no longer to submit new biddings, the whole bidding process is over, the auction reaches equilibrium state, and the equilibrium price is $p^{*}$ which can be computed by (8), the allocated quantity for bidder $j$ is $q_{j}^{*}$ which can be computed by (9). The bidder $j$ must pay $p^{*} q_{j}^{*}$ to get the quantity $q_{j}^{*}$.

\section{An application example}

In this section, we give an auction example to describe the forming process of equilibrium point.

Supposed that an auctioneer wants to auction $Q_{0}=150$ units of a continuous homogeneous divisible good (such as industrial coal, petroleum, emission rights, and so on). There are three bidders participate in multiple rounds bidding. The set of bidders is denoted as $N=\{1,2,3\}$. All bidders and the auctioneer are risk neutral. The maximum demand quantities of these three bidders are $G_{1}=110, G_{2}=70, G_{3}=100$ respectively, and the actual demand function of them are as follows:

$$
q_{1}^{(0)}(p)=150-7 p, q_{2}^{(0)}(p)=220-8 p, q_{3}^{(0)}(p)=120-8 p .
$$

Now suppose that bidder 3 has already submitted his actual demand function $q_{3}^{(1)}(p)=120-8 p$, i.e. bidder 3 no longer submits new bidding from the second round. The other two bidders' initial biddings are as follows:

$$
q_{1}^{(1)}(p)=120-9 p, q_{2}^{(1)}(p)=160-10 p
$$

By using model $M_{1}$ to allocate the total quantity $Q_{0}$. Substitute above relative data to $M_{1}$ and solve $M_{1}$, the uniform price is $p^{*}=9.26$, and the allocated quantities to bidders are $q_{1}^{*}=36.67, q_{2}^{*}=67.40, q_{3}^{*}=45.93$. And the actual total allocated quantity given by the auctioneer is $Q^{*}=150$, and his income is $U=1388.89$.

Although the scheduled supply $Q_{0}=150$ is full allocated to the bidders, the biddings of bidders 1 and bidder 2 don't reach the limit state, and don't realize maximization of allocated quantity. So the auction does not reach equilibrium state. The auctioneer will publish the current allocation results, and let bidders submit multiple rounds biddings according to the bidding rule of "Not allowed to modify downward rule".

Case 1: In the second round bidding, bidder 1 bids first, and bidder 2 follows bidder 1 .

Since the initial bidding of bidder 1 satisfies $a_{1}^{(0)}-b_{1}^{(0)} \cdot \frac{a_{1}^{(0)}+a_{2}^{(1)}+a_{3}^{(1)}-Q_{0}}{b_{1}^{(0)}+b_{2}^{(1)}+b_{3}^{(1)}}=71.6<G_{1}=110$, by Proposition 2, bidder 1 will choose his optimal strategy to maximize his income, that is, he 
will submit his actual demand function $q_{1}^{(2)}(p)=150-7 p$. Thus, the income of bidder 1 is maximal, and the current uniform price and allocated quantities are as follows:

$$
p^{*}=11.2, q_{1}^{*}=71.6, q_{2}^{*}=48, q_{3}^{*}=30.4 .
$$

And the actual total allocated quantity given by the auctioneer is $Q^{*}=150$, and his income is $U=1680$.

Then bidder 2 will submit his new biddings according to the current allocation results. Since $a_{2}^{(0)}-b_{2}^{(0)} \cdot \frac{a_{2}^{(0)}+a_{3}^{(1)}+a_{1}^{(2)}-Q_{0}}{b_{2}^{(0)}+b_{3}^{(1)}+b_{1}^{(2)}}=101.74>G_{2}=70$, by Proposition 2, bidder 2 will choose his optimal strategy to maximize his income, that is, $q_{2}^{(2)}(p)=a_{2}^{(2)}-b_{2}^{(2)} p$, where $a_{2}^{(2)}$ and $b_{2}^{(2)}$ satisfy:

$$
a_{2}^{(2)}-b_{2}^{(2)} \cdot \frac{a_{2}^{(2)}+a_{3}^{(1)}+a_{1}^{(2)}-Q_{0}}{b_{2}^{(2)}+b_{3}^{(1)}+b_{1}^{(2)}}=70 \text { and } 8 \leq b_{2}^{(2)} \leq 10 .
$$

When $b_{2}^{(2)}$ is determined, we can solve the value of $a_{2}^{(2)}$. For example, $a_{2}^{(2)}=184$ and $b_{2}^{(2)}=9, a_{2}^{(2)}=171.33$ and $b_{2}^{(2)}=8, a_{2}^{(2)}=190.33$ and $b_{2}^{(2)}=9.5$ are all the biddings which satisfy above two conditions. However, in these biddings, no matter what bidding bidder 2 submits, the final results are the same, i.e. the total supply $Q_{0}$ is full allocated to the bidders, and all bidders' incomes are no longer increased, and the auction reaches equilibrium state. The corresponding equilibrium point $\left(p^{*}, q_{1}^{*}, q_{2}^{*}, q_{3}^{*}\right)$ is as follows:

$$
p^{*}=12.67, q_{1}^{*}=61.33, q_{2}^{*}=70, q_{3}^{*}=18.67 .
$$

The auctioneer's actual income is $U=1900$.

Case 2: In the second round bidding, bidder 2 bids first, and bidder 1 follows bidder 2.

When bidder 2 bids first, since $a_{2}^{(0)}-b_{2}^{(0)} \cdot \frac{a_{2}^{(0)}+a_{3}^{(1)}+a_{1}^{(1)}-Q_{0}}{b_{2}^{(0)}+b_{3}^{(1)}+b_{1}^{(1)}}=120.8>G_{2}=70$, by Proposition 2, bidder 2 will choose his optimal strategy to maximize his income, that is, $q_{2}^{(2)}(p)=a_{2}^{(2)}-b_{2}^{(2)} p$, where $a_{2}^{(2)}$ and $b_{2}^{(2)}$ satisfy conditions $a_{2}^{(2)}-b_{2}^{(2)} \cdot \frac{a_{2}^{(2)}+a_{3}^{(1)}+a_{1}^{(1)}-Q_{0}}{b_{2}^{(2)}+b_{3}^{(1)}+b_{1}^{(1)}}=70$ and $8 \leq b_{2}^{(2)} \leq 10$, such as $a_{2}^{(2)}=159.41$ and $b_{2}^{(2)}=9.5, a_{2}^{(2)}=154.71$ and $b_{2}^{(2)}=9$, and so on.

However, in these biddings, no matter what bidding bidder 2 submits, the final results are the same, that is:

$$
p^{\star}=9.41, q_{1}^{*}=35.29, q_{2}^{*}=70, q_{3}^{*}=44.71 .
$$

And the actual total allocated quantity given by the auctioneer is $Q^{*}=150$, his actual income is $U=1411.76$.

Then bidder 1 will submit his new biddings according to the current allocation results. By the judgment method which is similar with case 1), we can determine that the optimal bidding strategy of bidder 1 is to submit his actual demand function $q_{1}^{(2)}(p)=150-7 p$, the corresponding allocation result is as follows:

$$
p^{*}=11.40, q_{1}^{*}=70.17, q_{2}^{*}=51.07, q_{3}^{*}=28.76 .
$$


When the second round bidding is over, obviously, the bidding of bidder 1 reaches the limit state, but the biddings of bidders 2 don't reach the limit state, and don't realize maximization of allocated quantity. So the auction does not reach equilibrium state, and bidder 2 will continue to submit new bidding in the third round bidding.

From the conclusion (iii) given in Proposition 2, the optimal bidding strategy of bidder 2 in the third round is the same as the optimal bidding strategy of bidder 2 in the second round. Meanwhile, the total supply $Q^{\star}=150$ is full allocated to the bidders, and all bidders' incomes are no longer increased, and the auction reaches equilibrium state. The corresponding equilibrium point $\left(p^{*}, q_{1}^{*}, q_{2}^{*}, q_{3}^{*}\right)$ is as follows:

$$
p^{*}=12.67, q_{1}^{*}=61.33, q_{2}^{*}=70, q_{3}^{*}=18.67 .
$$

The auctioneer's actual income is $U=1900$.

From the forming process of equilibrium point given by case 1) and case 2), we can conclude that bidders' bidding order is different, but the final equilibrium point is unique, in the other word, the final equilibrium point is independent of bidders' bidding order.

In fact, for above example, we can also prove that the final equilibrium point is independent of bidders' starting points of initial bidding. For example, we modify the initial biddings of three bidders $q_{1}^{(1)}(p)=120-9 p, q_{2}^{(1)}(p)=160-10 p, q_{3}^{(1)}(p)=120-8 p$ as new biddings $q_{1}^{(1)}(p)=130-9 p, q_{2}^{(1)}(p)=185-10 p, q_{3}^{(1)}(p)=120-8 p$. By use the optimal strategy given in Proposition 2, we can easily get the final equilibrium point in these new biddings is the same as the final equilibrium point given by case 1) and case 2).

Obviously, the result of equilibrium point is in accordance with the conclusion given by Proposition 2.

\section{Conclusions}

This paper studies the auction problem for a kind of continuous homogeneous divisible goods and presents a uniform price auction mechanism. Compared with the existing mechanisms of uniform price auctions, the contribution of the paper is as follows. In our mechanism, the bidders submit multiple rounds continuous linear bidding, all bidders are asymmetric, the auctioneer's supply is variable, and every bidder's valuation to per unit of the goods is independent private information. By exploring the bidders' asymptotic strategic behaviours and the forming process and composition of equilibrium points, the conclusion can be drawn that the different bidders' bidding order and different starting points of initial bidding would not cause different local equilibrium points, and if the equilibrium points exist, then the equilibrium point is unique. Therefore, the auction mechanism proposed in this paper can effectively improve the efficiency of resources allocation.

In future work we intend to analyse the effect of multi-attribute bidding strategy in more complex auction protocols such as multi-sourcing multi-attribute, combinatorial, double and simultaneous-multiple auctions. Moreover, as for other directions for our future research, we think the mechanism design for multi-dimensional auctions, two stages auctions in which the second stage includes negotiations, and how to apply these multi-attribute auctions to the fields of real business and industry world, etc. are valuable and interesting issues. 


\section{Acknowledgments}

This work is supported by the National Natural Science Foundation of China (No. 71201064, 61273206), the Project funded by China Postdoctoral Science Foundation (No. 2012M521432), the Special Fund of China Postdoctoral Science Foundation (No. 2013T60724), and the 2014 Key Project of Hubei Provincial Department of Education to C. J. Rao.

\section{References}

Back, K.; Zender, J. F. 1993. Auctions of divisible goods: on the rationale for the treasury experiment, Review of Financial Studies 6(1): 733-764. http://dx.doi.org/10.1093/rfs/6.4.733

Back, K.; Zender, J. F. 2001. Auctions of divisible goods with endogenous supply, Economics Letters 73(1): 29-34. http://dx.doi.org/10.1016/S0165-1765(01)00465-7

Bourjade, S. 2009. Strategic price discounting and rationing in uniform price auctions, Economics Letters 1: 23-27. http://dx.doi.org/10.1016/j.econlet.2009.05.008

Brenner, M.; Galai, D.; Sade, O. 2009. Sovereign debt auctions: uniform or discriminatory?, Journal of Monetary Economics 56(2): 267-274. http://dx.doi.org/10.1016/j.jmoneco.2008.12.012

Burinskiene, M.; Rudzkis, P. 2010. Feasibility of the liberal electricity market under conditions of a small and imperfect market. The case of Lithuania, Technological and Economic Development of Economy 16(3): 555-566. http://dx.doi.org/10.3846/tede.2010.34

Chakraborty, I.; Engelbrecht-Wiggans, R. 2005. Asymptotic prices in uniform-price multi-unit auctions summary, Economic Theory 26(4): 983-987. http://dx.doi.org/10.1007/s00199-004-0549-6

Damianov, D. S.; Becker, J. G. 2010. Auctions with variable supply: uniform price versus discriminatory, European Economic Review 54: 571-593. http://dx.doi.org/10.1016/j.euroecorev.2009.09.002

Damianov, D. S.; Oechssler, J.; Becker, J. G. 2010. Uniform vs. discriminatory auctions with variable supply-experimental evidence, Games and Economic Behavior 68: 60-76. http://dx.doi.org/10.1016/j.geb.2009.06.008

Feng, J.; Chatterjee, K. 2010. Simultaneous vs. sequential sales: bidder competition and supply uncertainty, Decision Support Systems 49: 251-260. http://dx.doi.org/10.1016/j.dss.2010.02.011

Genc, T. S. 2009. Discriminatory versus uniform-price electricity auctions with supply function equilibrium, Journal of Optimization Theory and Applications 140: 9-31. http://dx.doi.org/10.1007/s10957-008-9437-8

Groves, V. 2011. Charity auctions with multiple goods: bidding behavior and revenue, Economics Letters 111(2): 166-169. http://dx.doi.org/10.1016/j.econlet.2011.02.007

Holmberg, P. 2009. Supply function equilibria of pay-as-bid auctions derived equilibrium are compared to results for the SFE of a uniform-price auction, Journal of Regulatory Economics 36(2): 154-177. http://dx.doi.org/10.1007/s11149-009-9091-6

Holmberg, P.; Newbery, D. 2010. The supply function equilibrium and its policy implications for wholesale electricity auctions, Utilities Policy 18(4): 209-226. http://dx.doi.org/10.1016/j.jup.2010.06.001

Jain, R.; Walrand, J. 2010. An efficient Nash-implementation mechanism for network resource allocation, Automatica 46(8): 1276-1283. http://dx.doi.org/10.1016/j.automatica.2010.05.013

Klemperer, P. 1989. Supply function equilibria in oligopoly under uncertainty, Econometrica 57(6): 1243-1277. http://dx.doi.org/10.2307/1913707

Kremer, I.; Nyborg, K. 2004a. Divisible-good auctions: the role of allocation rules, Rand Journal of Economics 35(1): 147-159. http://dx.doi.org/10.2307/1593734 
116 C. Rao et al. Uniform price auction of divisible goods based on multiple rounds linear bidding ...

Kremer, I.; Nyborg, K. 2004b. Underpricing and market power in uniform price auctions, Review of Financial Studies 17(1): 849-877.

Lengwiler, Y. 1999. The multiple unit auction with variable supply, Economic Theory 14(2): 373-392. http://dx.doi.org/10.1007/s001990050299

LiCalzi, M.; Pavan, A. 2005. Tilting the supply schedule to enhance competition in uniform-price auctions, European Economic Review 49(1): 227-250. http://dx.doi.org/10.1016/S0014-2921(02)00324-0

Mezzetti, C.; Saša Pekeč, A.; Tsetlin, I. 2008. Sequential vs. single-round uniform-price auctions, Games and Economic Behavior 62(2): 591-609. http://dx.doi.org/10.1016/j.geb.2007.05.002

Milgrom, P. 2006. Putting auction theory to work. Beijing: Tsinghua University Press. 368 p.

McAdams, D. 2002. Modifying the uniform-price auction to eliminate "Collusive Seeming Equilibria", Working paper, MIT.

McAdams, D. 2007. Adjustable supply in uniform price auctions: non-commitment as a strategic tool, Economics Letters 95(1): 48-53. http://dx.doi.org/10.1016/j.econlet.2006.08.035

Kapliński, O.; Tamošaitiene, J. 2010. Game theory applications in construction engineering and management, Technological and Economic Development of Economy 16(2): 348-363. http://dx.doi.org/10.3846/tede.2010.22

Rao, C. J.; Peng, J. 2009. Fuzzy group decision making model based on credibility theory and gray relative degree, International Journal of Information Technology \& Decision Making 8(3): 515-527. http://dx.doi.org/10.1142/S0219622009003533

Rao, C. J.; Zhao, Y. 2010. Mechanism design for optimal auction of divisible goods, International Journal of Information Technology \& Decision Making 9(5): 831-845.

http://dx.doi.org/10.1142/S0219622010004044

Rao, C. J; Zhao, Y.; Wang, Q. 2010. Uniform price auctions of divisible goods with variable supply, ICIC Express letters 4(4): 1127-1134.

Rao, C. J.; Zhao, Y. 2011a. Optimal multi-attribute auctions for divisible goods, International Journal of Information Technology \& Decision Making 10(5): 891-911. http://dx.doi.org/10.1142/S0219622011004622

Rao, C. J.; Zhao, Y. 2011b. Incentive mechanism for allocating total permitted pollution discharge capacity and evaluating the validity of free allocation, Computers \& Mathematics with Applications 62(8): 3037-3047. http://dx.doi.org/10.1016/j.camwa.2011.08.015

Rao, C. J.; Zhao, Y.; Li, C. F. 2012. Asymmetric Nash equilibrium in emission rights auctions, Technological Forecasting and Social Change 79(3): 429-435. http://dx.doi.org/10.1016/j.techfore.2011.08.012

Rubchinsky, A. 2010. Brams-Taylor model of fair division for divisible and indivisible items, Mathematical Social Sciences 60(1): 1-14. http://dx.doi.org/10.1016/j.mathsocsci.2010.03.004

Sioshansi, R.; Nicholson, E. 2011. Towards equilibrium offers in unit commitment auctions with nonconvex costs, Journal of Regulatory Economics 40(1): 41-61. http://dx.doi.org/10.1007/s11149-011-9147-2

Wang, J. J. D.; Zender, J. F. 2002. Auctioning divisible goods, Economic Theory 19(3): 673-705. http://dx.doi.org/10.1007/s001990100191

Wilson, R. 1979. Auctions of share, The Quarterly Journal of Economics 93(4): 675-689. http://dx.doi.org/10.2307/1884475

Congjun RAO. He is currently an Associate Professor at the College of Mathematics and Computer Science of Huanggang Normal University. Received his BSc degree from Huanggang Normal University, and his MSc degree from Wuhan University of Technology, and his PhD degree from Huazhong University of Science and Technology. His main research interests include decision theory and methods, game theory, and auction theory. He has published more than 60 papers in various journals or conference proceedings. 
Yong ZHAO. He is currently a Professor at the Department of Control Science and Engineering of Huazhong University of Science and Technology. His BSc, MSc, and PhD degrees are from Huazhong University of Science and Technology and on Systems Engineering. He published many international journal papers and book chapters. His main research interests include decision theory and methods, engineering economics, and auction theory.

Yang CHEN. He is currently a Lecturer at the Department of Control Science and Engineering of Huazhong University of Science and Technology. His BSc, MSc, and PhD degrees are from Huazhong University of Science and Technology and on Systems Engineering. His main research interests include decision theory and methods, project management, developing data mining applications and other areas. 